Mini Review

\section{Physical activity can change the physiological and psychological circumstances during COVID-19 pandemic: A narrative review}

\author{
Khashayar Maroufi*
}

Faculty of Physical Education and Sport Sciences, University of Tehran, Tehran, Iran

\section{Abstract}

Background: With the outbreak of Coronavirus disease 2019 (COVID-19), many studies attention to this world's complexity increased dramatically. Different views on sports and physical activities have been presented, which have addressed the advantages and disadvantages of sports activities in this period differently. The purpose of this review was to investigate the physiological and psychological effects of physical activity during the COVID-19 pandemic.

Methods: Using PubMed, Science Direct, Medline, and Web of Science electronic databases, this review summarizes the current knowledge of direct and indirect effects of physical activity during the COVID-19 pandemic, evaluating the advantages and drawbacks of specific exercise physiology conditions. All types of studies were assessed, including systematic reviews, case-studies, and clinical guidelines. The literature search identified 40 articles that discussed COVID-19, immune system, the relation between immune system and exercise or diet, and psychological impacts of physical activity.

Results: Forty articles review showed that the immune system depends on the type, frequency, intensity, and duration of the exercise.

Intense or prolonged exercise with short recovery periods can progressively weaken the immune system and increase the risk of COVID-19. One of the acute responses after moderateintensity training is improved immune function and a decrease in inflammatory cytokines. Paying attention to dietary intakes of micro-and macronutrients in conjunction with exercise can strengthen the condition to fight against coronavirus. Exercise can also affect the psychological dimensions of the COVID-19 pandemic, including depression, anxiety, and stress, which improve community mental health during the quarantine.

Conclusion: Setting appropriate physical activity based on individuals' properties and proper diet plan may enhance the physiological and psychological body's condition to fight against coronavirus.

\section{More Information}

*Address for Correspondence: Khashayar Maroufi, Faculty of Physical Education and Sport Sciences, University of Tehran, Tehran, Iran, Tel: 00989124072520 ; Email: Khashayar.maroufi@ut.ac.ir

Submitted: January 15, 2021

Approved: January 25, 2021

Published: January 26, 2021

How to cite this article: Maroufi K. Physical activity can change the physiological and psychological circumstances during COVID-19 pandemic: A narrative review. J Sports Med Ther. 2021; 6: 001-007.

DOI: 10.29328/journal.jsmt. 1001051

ORCiD: orcid.org/0000-0002-2112-5270

Copyright: @ 2021 Maroufi K. This is an open access article distributed under the Creative Commons Attribution License, which permits unrestricted use, distribution, and reproduction in any medium, provided the original work is properly cited.

Keyword: COVID-19; Inflammation; Physical activity; Exercise physiology; Exercise psychology

Check for updates

OPEN ACCESS

\section{Introduction}

Coronavirus is one of the greatest human threats [1]. In the last few months, the increase in cases and deaths due to COVID-19 in the world and the imposition of more social restrictions in most countries have caused many physiological and psychological disorders [1,2]. Recorded data from smartwatches show that the level of physical activity worldwide during the COVID-19 pandemic decreased between $8 \%$ to $48 \%$ [3]. The elderly, obese, and sedentary individuals with a body mass index (BMI) of more than 25 $\mathrm{kg} / \mathrm{m} 2$ have been reported to be among the most vulnerable groups to the coronavirus [1]. According to a recently published study, patients with COVID-19 who are less than 60 years old and have a body mass index between 30 and 34 are 1.8 to 2 times more likely to be hospitalized than other people with a body mass index below $30[4,5]$. Also, lifestyle changes during this period and the stress caused by the disease can cause weight gain [6]. Although the exact mechanism for the increased risk of COVID-19 due to obesity is not clear, from the point of view of the respiratory system's function, it can be introduced through the enzyme angiotensin-converting enzyme (ACE2) as a coronavirus receptor. Obesity can Increase ACE2 levels and consequently escalate the risk of 
infection [7]. The mechanism of the immune system in the face of the coronavirus is that the virus is first detected in the patient's lungs and then attacked, which causes inflammation and eventually damage to the lung tissue. In the next stage, this injury disrupts the exchange of respiratory gases, which is more acute cases require serious medical intervention to save the patient's life In general, exercise has been shown to improve immune system function. Muscles secrete compounds during and after exercise to strengthen the immune system and reduce inflammation [8]. Although no studies have been performed on physical activity in patients with COVID-19, many studies have shown that exercise has beneficial effects on the immune system, inflammation, and respiratory system [9]. The type, amount, and intensity of an exercise determine the desired impact on the body's immune system and inflammation. Researches have shown that low to moderate-intensity exercise has far more significant effects on the immune system. Contrary, very intense and prolonged exercise, such as a Marathon, temporarily reduces the immune system, putting a person at risk for the disease [8]. Low- to moderate-intensity exercise can heighten the immune system by increasing glutamine and eventually regulating the function of macrophages, neutrophils, lymphocytes, and phagocytosis of some pathogens $[10,11]$.

Studies showed that the presence of some underlying diseases could increase the risk of more severe COVID -19 disease in individuals [12]. But many studies have illustrated that regular exercise can prevent many diseases of the respiratory, cardiovascular, metabolic, and cancers [13], which can be one of the advantages of exercise during the COVID -19 pandemic [8]. It has been reported, due to reduced physical activity of many segments of society, problems such as circadian rhythm disorders, energy intake, and reduced burned calories can increase the risk of infection and death due to this emerging disease [14].

The nutritional status of individuals has been considered as an indicator of resilience against diseases and destabilization. The ecology of adversity and resilience demonstrates that substantial stressors, such as inadequate nutrition, can lead to long-lasting effects linked to health [15]. When Ebola virus outbreak in Western Africa, researches showed that immediate supportive care significantly reduces case fatality rates. It may apply as well to the current COVID-19 pandemic [16]. It has been illustrated that an adequate diet can affect the immune system function through the activation of cells, modification in the production of signaling molecules, and gene expression [15]. Therefore, the purpose of this narrative review is to become more aware of the physiological and psychological impacts of training and adequate diet during the COVID-19 pandemic.

\section{Methods}

PubMed, Science Direct, Medline, and Web of science were searched to summarize the current knowledge of direct and indirect physiological and psychological effects of physical activity during the COVID-19 pandemic. A combination of the keywords "COVID-19," "physical activity," "training," "psychology," and "physiology" were investigated in the electronic databases. The literature search identified 40 articles in which nine articles considered COVID-19, fourteen articles investigated the immune system, nine articles evaluate effects of physical activities or diet on immune system function, and eight articles considered the relation between physical activity and psychological dimensions.

\section{Results}

There were some limitations about coronavirus and its interaction with physical activities. Up to now, no study has illustrated the straight effects of physical activity on patients with COVID-19. In general, all researches have considered the influences of physical activity on reducing the risks of COVID-19 [1,17-20]. Studies, before the occurrence of the COVID-19 pandemic, had examined the relationship between exercising and the immune system function $[21,22]$. Moreover, it can be expanded to the effects of exercise on psychological dimensions, including anxiety, depression, and stress. As the same, no study has been found that had investigated the psychological effects of physical activity on confirmed or survived patients with COVID-19. Several studies have reported only socio-demographic about patients with COVID-19 that no intervention was executed, which causes exclusion from the present study [23]. In the following, some outcomes of physical activity on the physiological and psychological dimensions have been discussed.

\section{Physiological effects of physical activity during the COVID-19 pandemic}

In general, metabolic diseases such as hypertension, diabetes, insulin resistance, dyslipidemia, obesity, respiratory and cardiovascular diseases, and liver and kidney diseases increase the risk of COVID-19. But exercising can pave the way to lose weight, improve respiratory system function, regulate hormone homeostasis, and reduce inflammation [1,24]. Age, underlying medical condition, and obesity have been common among people infected with or killed by COVID-19 [4]. Researches have illustrated that regular exercise in the elderly can heighten the immune system. However, some exercise protocols are not possible due to joint and muscle injuries in the elderly. Many studies have examined the acute effects of an exercise bout on the immune system of the elderly, and the results showed that even one session could improve immune response [25]. However, studies on athletes and their immune system responses in short-term and longterm training demonstrated that the immune system is enhanced by regular exercise, suggesting that athletes may be more resistant to the coronavirus than others[17]. On the other hand, little information is available on the longterm effects of training on the immune system of the elderly 
[26]. Obese people are also more susceptible to COVID-19 due to the presence of the ACE2 enzyme. ACE2 is one of the receptors for the coronavirus $[1,27]$; therefore, increasing the calories burned during exercise can lead to weight loss and thus reduce the risk of COVID-19. Physical activity triggers muscle and liver glycogen breakdown, which prepares the breakdown of fat in adipose and muscle tissue and ultimately causes weight loss [28]. Previous studies have explained that exercise, regardless of its type, significantly reduces fat mass in obese people [29]. Studies have shown that respiratory and cardiovascular fitness is inversely related to obesity; hence, healthier respiratory and cardiovascular fitness in the normal weight range might be a powerful defensive tool to fight against coronavirus [1,27]. Exercise can improve the function of the respiratory system by raising the supply of oxygen and the uptake of oxygen by the muscles. In a study conducted by Church, et al. [30] on men between the ages of 45 and 75, they found that their oxygen consumption increased significantly after six months of regular aerobic exercise, indicating an improvement in the respiratory system. In another study, Church, et al. [31] reported that the respiratory function of women and men with inadequate respiratory systems has significantly increased with the implementation of endurance or resistance exercises.

According to clinical reports, Cytokines such as Interleukin-2 (IL-2), Interleukin-7 (IL-7), Interleukin-10 (IL-10), Granulocyte Colony-stimulating Factor (G-CSF), Interferongamma Inducible Protein (IP-10), Monocyte Chemoattractant Protein 1 (MCP-1), Macrophage Inflammatory Protein (MIP1A), and Tumour Necrosis Factor alpha (TNF $\alpha$ ) are increased in patients with COVID-19. Activation and spread of these cytokines lead to inflammation and endothelial dysfunction; eventually, these factors will cause problems for the body's arteries [3,32].

Likewise, lifestyle has been modified due to quarantine [1]. Insufficient physical activity develops the incidence of venous thromboembolism, which leads to consequences, such as cardiovascular problems and, in some cases, death [3]. Researchers have announced an increase in venous thromboembolism in confirmed COVID-19 patients can progress mortality [33]. Thus, it has been recommended that regular physical activity strengthens the immune system and improves the homeostasis conditions [1,3].

\section{Type, frequency, volume, and intensity of exercise}

Several factors identify an Exercise. Type, frequency, volume, and intensity are the remarkable variables that must be considered when individuals want to set their goals. These targets varied from case to case, including losing fat, building muscle, or enhancing performance $[1,34,35]$. Based on the American Sports Medicine Association (ACSM) guidelines, performing at least 150 minutes a week of moderate-intensity aerobic exercise is necessary to maintain public health [36]. According to a guide published by the World Health
Organization (WHO) in 2019, individuals should have five sessions per week for 30 to 60 minutes of regular moderate to high-intensity exercise [1]. According to the ACSM Guidelines on aerobic exercise, people who want to lose weight should spend more time per week on regular exercise. The ACSM has published standards on physical activity and its effects on weight loss in adults, as shown in table 1 [34].

Research has shown that even one exercise bout can provide the desired response to improve immune system function. Still, regular exercise more than once can have far more significant benefits for individuals [19]. According to previous research, only strenuous, prolonged activities reduce the immune system and increases the risk of infection, which during the COVID-19 pandemic can increase the risk of COVID-19 [19,22]. Many researchers have modeled these relationships with the letters J and S curves (Figure 1) [37].

Some researchers have also used the term "open window" after strenuous exercise. When the intensity, duration, and frequency of strenuous activities increase without a proper recovery period, the immune system will respond to viruses and infections for three to 72 hours that can expose an individual to COVID-19 (Figure 2) [18,38].

Table 1: Appropriate physical activity intervention strategies for weight loss and prevention of weight regain for adults.

\begin{tabular}{|c|c|}
\hline Weight Loss & Weight Loss \\
\hline No significant weight loss & $>150$ min.week $^{-1}$ \\
\hline $2-3 \mathrm{~kg}$ week $^{-1}$ & $150-225$ min.week $^{-1}$ \\
\hline $5-7.5 \mathrm{~kg} \cdot$ week $^{-1}$ & $225-420$ min.week $^{-1}$ \\
\hline Weight stabilization after a period of weight loss & $200-300$ min.week $^{-1}$ \\
\hline
\end{tabular}
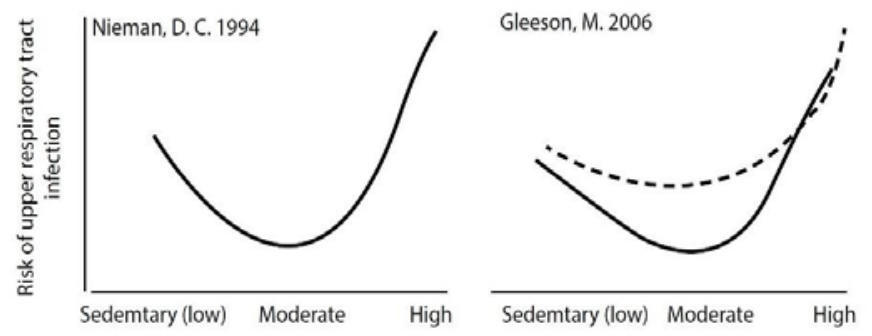

Exercise training load and volume

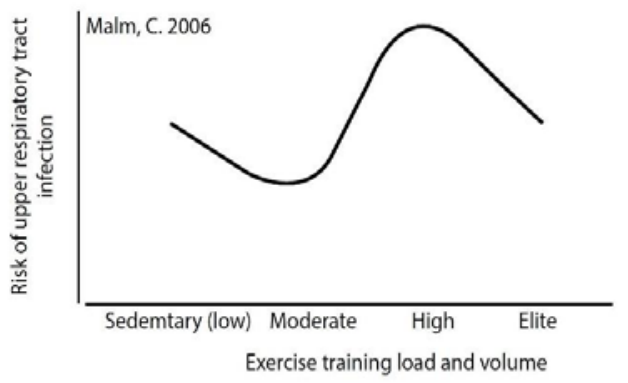

Figure 1: The two J-shaped curves (Nieman, 1994; Glesson, 2006) show the effect of exercise load and volume on upper respiratory tract infection risk. The S-shaped curve (Malm, 2006) also indicates that elite athletes are an exception to this rule. By increasing the load and volume of training, upper respiratory tract infection risk will be decreased [37]. 


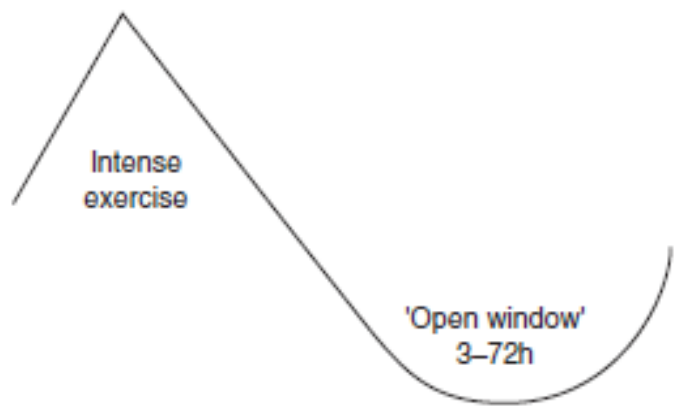

b

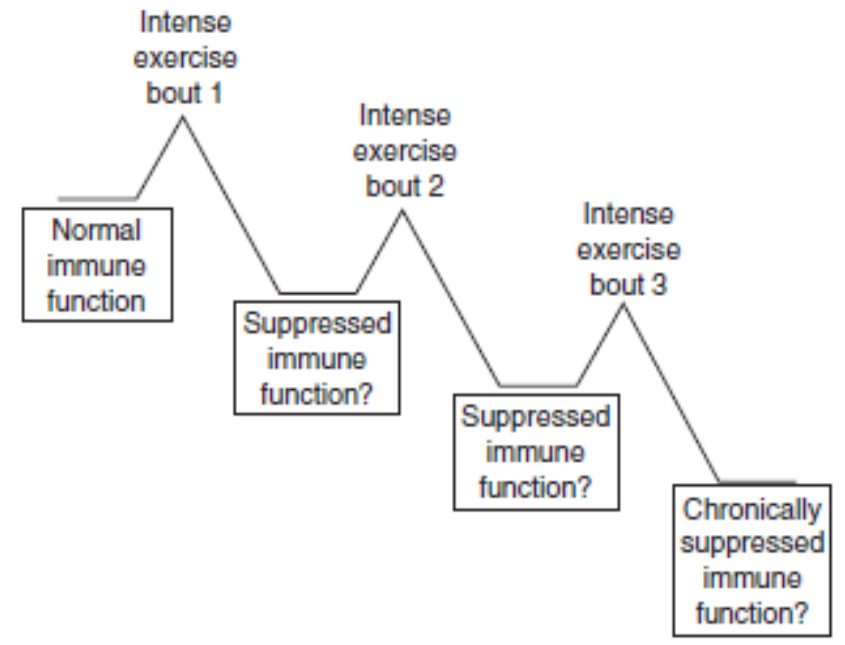

Figure 2: a) The open window occurs between three to 72 hours after the exercise, which exposes the individual to illness and infection. b) Shows the effect of training sessions on the immune system without considering the appropriate recovery period [1].

Resistance and High-intensity interval training (HIIT), such as CrossFit [35], must be performed at a certain intensity, frequency, and volume. Resistance training and HIIT usually involve periods of effort from a few seconds to a few minutes [39]. Notably, resistance training and HIIT are not directly recommended for weight loss, and it is suggested that diet and aerobic exercise should be utilized for weight loss [1]. Intensity, volume, and frequency of resistance workout or HIIT have to be adjusted according to the individual's readiness level to prevent any infection, inflammation, and ultimately reduced immune function during the COVID-19 pandemic [19,22,37].

\section{Diet}

It is recommended to use macro-and micronutrients in an adequate amount and in proportion to exercise duration and intensity to maintain the immune system following exercise. However, some reports suggested that many athletes use unhealthy diets high in sugar and fat due to restrictions and quarantine, impairing immune function. Studies have indicated that fasting can reduce some inflammatory factors, oxidative stress, reactive protein C (CRP), and IL-6 [20]. Based on the previous studies, the essential notion of the association between dietary components, nutrition, inflammation, and oxidative stress is well-regarded. It has been emphasized, for example, in the development of the anti-inflammatory dietary index [40]. Hence, paying attention to the microand macronutrients plays a pivotal role in reducing the risk of catching COVID-19. Anti-inflammatory and antioxidants ingredients, for instance, Omega-3 fatty acids, Vitamin A, Vitamin C, can be found in plant-based foods $[6,40]$. Low protein intake can elevate the risk of infection, and for athletes, it can trigger inappropriate consequences including, performance reduction and losing muscle mass [15,41]. Moreover, consumed calories must be adjusted according to intensity, volume, and frequency of exercise and physiological conditions to increase the immune system>s optimal function and reduce the open window>s time to some extent $[20,21]$.

Among micronutrients, Vitamin D is highly recommended as an essential nutrient that enters the body through the sun's ultraviolet rays or some food sources such as fish and grains, egg yolks, and dried mushrooms [42]. An adequate supply of zinc, selenium, and vitamin D has been suggested as the essential part for resistance to other viral infections, immune function, and reduced inflammation [43]. Experts have reported a significant correlation between vitamin D deficiency and the incidence of acute respiratory infections, especially in patients with COVID-19 [20]. In general, vitamin D inhibits the production of IL- 1 and TNF $\alpha$ by monocytes; hence, vitamin D may play an essential role in reducing the risk of COVID-19. 10,000 IU of vitamin D per day for several consecutive weeks is reported as an effective dose [44]. Although many studies investigated vitamin D worldwide, only a few studies have measured this vitamin intake in athletes. Researches demonstrated that athletes in different regions have different levels of vitamin $D$ and factors, such as training seasons (summer or winter), training hours, geographical location, training environment (indoors or outdoors), and use of skin protection [42]. Therefore, due to the restrictions and quarantine and the absence of people in the outdoor environment and not facing sunlight, the use of a diet rich in vitamin D can raise immune system function [20].

\section{Psychological effects of physical activity during the COVID-19 pandemic}

Severe restrictions and quarantine in some countries due to coronavirus outbreaks have led to an escalation in mental disorders, such as depression, anxiety, and stress. Although some may find quarantine discriminatory and offensive, quarantine is used as a practical solution in communities to control coronavirus spread [45]. Previous researches have shown that the widespread prevalence of infectious and epidemic diseases is associated with an increased majority of psychological difficulties. Results show that the COVID-19 pandemic has been one factor responsible for psychological distress in different population segments [46]. Moreover, the pandemic has caused the occurrence of sleep disorders. Therefore, it is reasonable to speculate that psychological conditions may be compromised during the COVID-19 outbreak, not only in the population directly affected by the virus but also in the general population [47]. 
Many studies have emphasized that regular exercise can alter the prevention and treatment of anxiety and depression [47-49]. Anxiety and depression, despite psychological effects, may cause a tendency to passivity and withdrawal, which has unpleasant consequences related to physiological markers during the pandemic $[1,48]$. Randomized controlled studies have confirmed that exercise is associated with an anti-depressant effect [48]. A meta-analysis reported, using the Hamilton rating scale for depression, regular exercise, and physical activity can treat individuals who were diagnosed with clinical depression or among those who do not respond to medication [50]. A study demonstrated that three weeks of a full-body workout, 45 min each session, by women with mild to moderate clinical depression could improve their mental health status [51]. Moreover, the idea that exercise can only change individuals' condition with depression is unreliable because results showed that regular exercise and physical activity might reduce depression and anxiety in non-clinical populations $[48,50]$.

According to preceding studies, nearly one-half of people diagnosed with depression will also experience comorbid anxiety. Anxiety contributes to emotional changes, sleep disorders, and poor concentration $[2,23,47]$. One study found that people who did not have access to gyms during the COVID-19 pandemic could reduce their anxiety using a combination of exercise and digital games. This game diminished the level of anxiety in healthy people for 20 minutes with moderate intensity. By performing it for 60 minutes two days per week for a total of eight weeks, it was able to reduce the level of anxiety in patients $[2,48]$.

Researchers have illustrated that regular physical activity by positively regulating brain-derived neurotrophic factor (BDNF) in the hippocampus, the main center of learning and memory, increases cognitive function and increases mice's ability to cope with depression and anxiety $[2,52]$. This mechanism may be defined by the accumulation of an endogenous molecule, D- $\beta$-hydroxybutyrate (DBHB), in the hippocampus, which can be elevated after long-term exercise and cross the blood brain barriers and improve the expression of BDNF [2].

Numerous studies have used aerobic training to examine the effect of exercise on psychological aspects. However, it has been shown that resistance exercise, or HIIT, effectively reduces psychological disorders. The intensity and type of physical activity recommended by the ACSM for weight loss and WHO for health are also advised to improve life quality, manage stress, and reduce depression and anxiety [49]. There is evidence that even low amounts (frequency, duration, and intensity) of physical activity protect against depression [50].

\section{Discussion}

The ongoing epidemic has been declared by the World Health Organization (WHO) as a global public health emergency[15]. According to preceding and current researches, regular physical activity can change coping with COVID-19, and attention to factors, such as individual differences, experience, and type of sports, play a crucial role. Regular exercise and physical activity can significantly enhance the immune system. Physical activity acts as a doubleedged sword because by increasing its intensity and duration, the function of the immune system is violated and provides the preconditions of COVID-19 infection. "Open window" is a crucial concept for setting the amount of physical activity and exercise [38]. Three to 72 hours after exercising, the immune system may be susceptible, which exposes individuals to illness and infection. The influence of regular exercise on weight loss is another benefit of reducing the risk of COVID-19. According to the ACSM guideline, at least 150 min.week-1 physical activity is needed for individuals' health. Moreover, for losing weight and improving physical readiness, more than 150 min.week-1 is recommended [34,37].

Nutrition intake is another remarkable variable to fight against coronavirus. Using a diet with sufficient microand macronutrients in the desired sports direction is a robust solution [20]. For instance, Anti-inflammatory and antioxidants ingredients can enhance the immune system. Contrary, Low protein diet may increase the risk of COVID-19. This type of diet may cause problems for athletes, such as losing muscle due to a reduction in protein synthesis [41]. In many studies, Vitamin D is recommended as an essential nutrient; however, due to the restrictions and quarantine and the absence of people in the outdoor environment and not facing sunlight, the use of a diet rich in vitamin D can heighten the immune system function. The intake of calories in conjunction with exercise should be considered during the COVID-19 pandemic. Losing weight and enhancing the immune system depends on the daily calorie intake.

Another advantage of exercise is overcoming the psychological problems caused by quarantine, such as depression, anxiety, and stress. Regular exercise improves one's self-esteem and a sense of well-being [53]. By considering ACSM and WHO guidelines, individuals can improve their psychological condition and prevent some of these disorders during COVID-19 pandemic. Many investigations are needed to investigate the effects of physical activity on COVID-19 patients in the future. In conclusion, it is crucial to consider the impact of lifestyle habits, including attention to the regular exercises and consumption of adequate and healthy microand macronutrients to prevent catching COVID-19.

\section{References}

1. Wang M, Baker JS, Quan W, Shen S, Fekete G, et al. A preventive role of exercise across the coronavirus 2 (SARS-CoV-2) pandemic. Front Physiol. 2020; 11: 572718.

PubMed: https://www.ncbi.nlm.nih.gov/pmc/articles/PMC7506115/

2. Hu S, Tucker L, Wu C, Yang L. Beneficial effects of exercise on depression and anxiety during the Covid-19 pandemic: A narrative review. Front Psychiatry. 2020; 11: 1217.

PubMed: https://pubmed.ncbi.nlm.nih.gov/33329133/ 
3. Zadow EK, Wundersitz DWT, Hughes DL, Adams MJ, Kingsley MIC et al., editors. Coronavirus (COVID-19), Coagulation, and Exercise: Interactions That May Influence Health Outcomes. Semin Thromb Hemost. 2020; 46: 807-814.

PubMed: https://pubmed.ncbi.nlm.nih.gov/32882720/

4. Chiappetta S, Sharma AM, Bottino V, Stier C. COVID-19 and the role of chronic inflammation in patients with obesity. International J Obes. 2020:1-3.

5. Kalligeros M, Shehadeh F, Mylona EK, Benitez G, Beckwith CG, et al. Association of obesity with disease severity among patients with coronavirus disease 2019. Obesity. 2020; 28: 1200-1204.

PubMed: https://pubmed.ncbi.nlm.nih.gov/32352637/

6. Mattioli AV, Sciomer S, Cocchi C, Maffei S, Gallina S. Quarantine during COVID-19 outbreak: changes in diet and physical activity increase the risk of cardiovascular disease. Nutr Metab Cardiovasc Dis. 2020; 30: 1409-1417.

PubMed: https://pubmed.ncbi.nlm.nih.gov/32571612/

7. Nishimura $\mathrm{H}$, Itamura S, Iwasaki T, Kurata T, Tashiro M. Characterization of human influenza A (H5N1) virus infection in mice: neuro-, pneumoand adipotropic infection. J Gen Virol. 2000; 81: 2503-2510.

PubMed: https://pubmed.ncbi.nlm.nih.gov/10993940/

8. Sallis JF, Pratt M, Sallis JF. Multiple benefits of physical activity during the Coronavirus pandemic. Rev Bras Ativ Fís Saúde. 2020; 25: 1-5.

9. Meneses-Echávez JF, Correa-Bautista JE, González-Jiménez E, RíoValle JS, Elkins MR, et al. The effect of exercise training on mediators of inflammation in breast cancer survivors: a systematic review with metaanalysis. Cancer Epidemiol Biomarkers Prev. 2016; 25: 1009-1017. PubMed: https://pubmed.ncbi.nlm.nih.gov/27197276/

10. Neto JR, Lira F, De Mello M, Santos RVT. Importance of exercise immunology in health promotion. Amino Acids. 2011; 41: 1165-1172. PubMed: https://pubmed.ncbi.nlm.nih.gov/20976509/

11. Kizaki T, Takemasa T, Sakurai T, Izawa T, Hanawa T, et al. Adaptation of macrophages to exercise training improves innate immunity. Biochem Biophys Res Commun. 2008; 372: 152-156.

PubMed: https://pubmed.ncbi.nlm.nih.gov/18474227/

12. Henry BM, De Oliveira MHS, Benoit S, Plebani M, Lippi G. Hematologic, biochemical and immune biomarker abnormalities associated with severe illness and mortality in coronavirus disease 2019 (COVID-19): a meta-analysis. Clin Chem Lab Med. 2020; 58: 1021-1028.

PubMed: https://pubmed.ncbi.nlm.nih.gov/32286245/

13. Powell KE, King AC, Buchner DM, Campbell WW, DiPietro L, et al. The Scientific Foundation for the Physical Activity. J Phys Act Health. 2019; 16: 1-11.

PubMed: https://pubmed.ncbi.nlm.nih.gov/30558473/

14. Molanouri Shamsi M, Amani Shalamzari S. Exercise Training, Immune System, and Coronavirus. Sport Physiology. 2020; 12: 17-40.

15. Naja F, Hamadeh R. Nutrition amid the COVID-19 pandemic: a multilevel framework for action. Eur J Clin Nutr. 2020; 74: 1117-1121. PubMed: https://pubmed.ncbi.nlm.nih.gov/32313188/

16. Laviano A, Koverech A, Zanetti M. Nutrition support in the time of SARS-CoV-2 (COVID-19). Nutrition. 2020; 74: 110834.

PubMed: https://pubmed.ncbi.nlm.nih.gov/32276799/

17. Letieri RV, Furtado GE. Physical exercise during coronavirus disease (COVID-19): Recommendations to remaining active in periods of confinement. Anais da Academia Brasileira de Ciências. 2020; 92.

18. Shi Y, Wang Y, Shao C, Huang J, Gan J, et al. COVID-19 infection: the perspectives on immune responses. Nature Publishing Group; 2020. PubMed: https://pubmed.ncbi.nlm.nih.gov/32205856/

19. Shirvani $H$, Rostamkhani $F$. Exercise considerations during coronavirus disease 2019 (COVID-19) Outbreak: A narrative review. J Military Med. 2020; 22: 161-168.
20. Yousfi N, Bragazzi NL, Briki W, Zmijewski P, Chamari K. The COVID-19 pandemic: how to maintain a healthy immune system during the lockdown-a multidisciplinary approach with special focus on athletes. Biol Sport. 2020; 37: 211-216.

PubMed: https://pubmed.ncbi.nlm.nih.gov/32879542/

21. Gleeson M, Nieman DC, Pedersen BK. Exercise, nutrition and immune function. J Sports Sci. 2004; 22: 115-125.

PubMed: https://pubmed.ncbi.nlm.nih.gov/14971437/

22. Pedersen BK, Bruunsgaard $H$, Jensen $M$, Toft $A D$, Hansen $H$, et al Exercise and the immune system-influence of nutrition and ageing. $J$ Sci Med Sport. 1999; 2: 234-252.

23. Verma S, Mishra A. Depression, anxiety, and stress and sociodemographic correlates among general Indian public during COVID-19. Int J Soc Psychiatry. 2020; 66: 756-762.

PubMed: https://pubmed.ncbi.nlm.nih.gov/32567466/

24. Kodama S, Saito K, Tanaka S, Maki M, Yachi Y, et al. Cardiorespiratory fitness as a quantitative predictor of all-cause mortality and cardiovascular events in healthy men and women: a meta-analysis. JAMA. 2009; 301: 2024-2035.

PubMed: https://pubmed.ncbi.nlm.nih.gov/19454641/

25. Simpson RJ, Cosgrove C, Ingram LA, Florida-James GD, Whyte GP, et al. Senescent T-lymphocytes are mobilised into the peripheral blood compartment in young and older humans after exhaustive exercise. Brain Behavior Immunity. 2008; 22: 544-551.

26. Simpson RJ, Lowder TW, Spielmann G, Bigley AB, LaVoy EC, et al. Exercise and the aging immune system. Ageing Res Rev. 2012; 11 404-420.

PubMed: https://pubmed.ncbi.nlm.nih.gov/22465452/

27. Chan KK, Dorosky D, Sharma P, Abbasi SA, Dye JM, et al. Engineering human ACE2 to optimize binding to the spike protein of SARS coronavirus 2. Science. 2020; 369: 1261-1265. PubMed: https://pubmed.ncbi.nlm.nih.gov/32753553/

28. Poirier $P$, Després JP. Exercise in weight management of obesity. Cardiol Clin. 2001; 19: 459-470.

PubMed: https://pubmed.ncbi.nlm.nih.gov/11570117/

29. Martin CK, Johnson WD, Myers CA, Apolzan JW, Earnest CP, et al. Effect of different doses of supervised exercise on food intake, metabolism, and non-exercise physical activity: The E-MECHANIC randomized controlled trial. Am J Clini Nutr. 2019; 110: 583-592. PubMed: https://pubmed.ncbi.nIm.nih.gov/31172175/

30. Church TS, Earnest CP, Skinner JS, Blair SN. Effects of different doses of physical activity on cardiorespiratory fitness among sedentary, overweight or obese postmenopausal women with elevated blood pressure: a randomized controlled trial. JAMA. 2007; 297: 2081-2091. PubMed: https://pubmed.ncbi.nlm.nih.gov/17507344/

31. Church TS, Earnest CP, Thompson AM, Priest E, Rodarte RQ, et al. Exercise without weight loss does not reduce $C$-reactive protein: the INFLAME study. Med Sci Sports Exerc. 2010; 42: 708-716. PubMed: https://pubmed.ncbi.nlm.nih.gov/19952828/

32. Gaertner F, Massberg S, editors. Blood coagulation in immunothrombosis-At the frontline of intravascular immunity. Semin Immunol. 2016; 28: 561-569.

PubMed: https://pubmed.ncbi.nlm.nih.gov/27866916/

33. Lippi G, Sanchis-Gomar F, Henry BM. Coronavirus disease 2019 (COVID-19): the portrait of a perfect storm. Ann TransI Med. 2020; 8: 497. PubMed: https://pubmed.ncbi.nlm.nih.gov/32395541/

34. Swift DL, McGee JE, Earnest CP, Carlisle E, Nygard M, et al. The effects of exercise and physical activity on weight loss and maintenance. Prog Cardiovasc Dis. 2018; 61: 206-213.

PubMed: https://pubmed.ncbi.nlm.nih.gov/30003901/

35. Maroufi K, Razavi R, Gaeini AA, Nourshahi M. The effects of acute consumption of carbohydrate-protein supplement in varied ratios on CrossFit athletes' performance in two CrossFit exercises: a randomized 
cross-over trial. J Sports Med Phys Fitness. 2020.

PubMed: https://pubmed.ncbi.nlm.nih.gov/33314886/

36. Bushman B, Medicine ACoS. ACSM's Complete Guide to Fitness \& Health, 2E: Human Kinetics; 2017.

37. Camargo-Junior F, Chalhub T, Moraes Filho JMd, Santos R, Feitosa F, et al. Covid-19 and its effect on olympic sport: the importance of studying social isolation and the harm it causes, in order to minimize it. Revista Brasileira de Medicina do Esporte. 2020; 26: 371-377.

38. Smith LL. Overtraining, excessive exercise, and altered immunity. Sports Med. 2003; 33: 347-364.

PubMed: https://pubmed.ncbi.nlm.nih.gov/12696983/

39. Xie B, Yan X, Cai X, Li J. Effects of high-intensity interval training on aerobic capacity in cardiac patients: a systematic review with metaanalysis. BioMed Res Int. 2017; 2017.

PubMed: https://pubmed.ncbi.nlm.nih.gov/28386556/

40. Iddir M, Brito A, Dingeo G, Fernandez Del Campo SS, Samouda H, et al. Strengthening the immune system and reducing inflammation and oxidative stress through diet and nutrition: considerations during the COVID-19 crisis. Nutrients. 2020; 12: 1562.

PubMed: https://pubmed.ncbi.nlm.nih.gov/32471251/

41. Venkatraman JT, Pendergast DR. Effect of dietary intake on immune function in athletes. Sports Med. 2002; 32: 323-337.

PubMed: https://pubmed.ncbi.nlm.nih.gov/11929359/

42. Larson-Meyer DE, Willis KS. Vitamin D and athletes. Curr Sports Med Rep. 2010; 9: 220-226.

PubMed: https://pubmed.ncbi.nlm.nih.gov/20622540/

43. Alexander J, Tinkov A, Strand TA, Alehagen U, Skalny A, et al. Early nutritional interventions with zinc, selenium and vitamin $D$ for raising antiviral resistance against progressive COVID-19. Nutrients. 2020; 12: 2358. PubMed: https://pubmed.ncbi.nlm.nih.gov/32784601/

44. Grant WB, Lahore H, McDonnell SL, Baggerly CA, French CB, et al. Evidence that vitamin $D$ supplementation could reduce risk of influenza and COVID-19 infections and deaths. Nutrients. 2020; 12: 988. PubMed: https://pubmed.ncbi.nIm.nih.gov/32252338/
45. de Lima CVC, Cândido EL, da Silva JA, Albuquerque LV, de Menezes Soares $\mathrm{L}$, et al. Effects of quarantine on mental health of populations affected by Covid-19. Journal of affective disorders. 2020; 275: 253-254. PubMed: https://pubmed.ncbi.nlm.nih.gov/32734916/

46. Li W, Yang Y, Liu ZH, Zhao YJ, Zhang Q, et al. Progression of mental health services during the COVID-19 outbreak in China. Int J Biol Sci. 2020; 16: 1732-1738.

PubMed: https://www.ncbi.nlm.nih.gov/pmc/articles/PMC7098037/

47. Casagrande M, Favieri F, Tambelli R, Forte G. The enemy who sealed the world: Effects quarantine due to the COVID-19 on sleep quality, anxiety, and psychological distress in the Italian population. Sleep Med. 2020; 75: 12-20.

PubMed: https://pubmed.ncbi.nlm.nih.gov/32853913/

48. Martinsen EW. Physical activity in the prevention and treatment of anxiety and depression. Nord J Psychiatry. 2008; 62(sup47): 25-29. PubMed: https://pubmed.ncbi.nlm.nih.gov/18752115/

49. Conn VS. Anxiety outcomes after physical activity interventions: metaanalysis findings. Nurs Res. 2010; 59: 224-231. PubMed: https://pubmed.ncbi.nlm.nih.gov/20410849/

50. Nagata S, McCormick BP, Austin DR. Physical Activity as Treatment for Depression in Recreation Therapy: Transitioning from Research to Practice. Therapeu Recreat J. 2020; 54.

51. Divya Mary S, Kirupa K, Vaishnavi G, Yamuna K, Revathi $P$, et al. Effects of total body workout in women with mild-to-moderate clinical depression. Drug Invention Today. 2019; 11.

52. Vosadi E, Ravasi AA, Choobine S, Barzegar H, Borjianfard M. Effect of endurance training and omega-3 supplementation in brain-derived neurotrophic factor (BDNF) in male adult rat hippocampus. Razi J Med Sci. 2013; 20: 50-57.

53. Maugeri G, Castrogiovanni P, Battaglia G, Pippi R, D'Agata V, et al. The impact of physical activity on psychological health during Covid-19 pandemic in Italy. Heliyon. 2020; 6: e04315.

PubMed: https://www.ncbi.nlm.nih.gov/pmc/articles/PMC7311901/ 